\section{Medicine's gender pay gap is still a glaring manifestation of pervasive inequality}

\author{
Latifa Patel, David Wrigley
}

The theme of this year's International Women's Day is the hope of a world free of bias, stereotypes, and discrimination, but the current reality is that inequality is still all pervasive. These deeply embedded cultural problems that exist within many areas of society, including medicine, disproportionately affect the career progression of women and the increasing gender pay gap is one manifestation of this. ${ }^{1}$

In our 2021 report on sexism in medicine, ${ }^{2}$ we heard from more than 2000 doctors, with $91 \%$ of women respondents saying they had experienced sexism at work, and 74\% saying they felt that this acted as a barrier to career progression. When it comes to tackling the gender pay gap, it's this widespread sexism that's compounding the problem.

Today on International Women's Day we are presenting some of our findings and our recommendations to the Department of Health and Social Care's (DHSC's) Gender Pay Gap Implementation Panel to help find a way through and to tackle this problem head on.

Gender equality is a huge priority for our membership and, since the publication of the gender pay gap in medicine review, ${ }^{3}$ we've been collecting evidence and listening to our members' experiences to gain a better understanding of what the barriers are to achieving gender equality in medicine. We recognise that change needs to occur at a societal, institutional, and interpersonal level, but we will continue to push where we have some influence.

The past few years have seen some notable wins worth celebrating. The new contract for specialty and associate specialist (SAS) doctors secured a flatter pay scale that will help with the gender pay gap and the new SAS senior grade gives senior specialist doctors the recognition they deserve. We've also seen the establishment of the DHSC's Gender Pay Gap Implementation Panel, which has agreed to analyse medical workforce data at a granular level to fully understand disparities in gender pay and invited the BMA to join as a member.

Yet it is important to highlight some of the areas that we believe must be prioritised now:

Pregnancy, return to work, and women's health are fundamental areas of concern and the current plans from the DHSC's Gender Pay Gap Implementation Panel don't give enough support to doctors. Among our recommendations is building a compassionate culture to ensure that women don't fall even further behind in their career progression. Childcare provision must be increased, but it needs to be affordable because women already shoulder disproportionate amounts of financial and career costs compared to men. We urge the panel to decide whether NHS nurseries will be introduced or a subsidy provided.

Enhanced shared parental leave and maternity leave must be available for all doctors and not used as a negotiating tool. Changing the culture where traditionally women are the ones to alter their working patterns for childcare purposes is fundamental to addressing gender disparities, and equity of access to enhanced shared parental leave is important to prevent the continued gendered perceptions of different specialties.

Doctors with multiple protected characteristics can face multiple forms of disadvantage and discrimination, compounding gender inequality. We'd like to see employers systematically reporting data on this, for all specialties.

Training pathways need to be more flexible and more accommodating for doctors who take periods of leave, work less than full time, or have career breaks. But the only way to tackle this is to increase the number of training places across the UK and across specialties. The shortage of trainees undermines flexible working because it results in staff shortages. With limited staff available, employers are less willing to allow flexible work and training.

Pay scales and the gender pension gap disadvantage women. If you have breaks in your service, or work less than full time, your pension is affected, and your ability to move up the pay scales is limited.

It's been two years since the first gender pay gap in medicine review, and we are now six months into the implementation panel's one year term. The BMA has established a Gender Pay Gap Working Group and consulted with doctors and it's their voices which have shaped these insights. We recommend that this and review overall progress, and for DHSC to provide adequate resources with measurable actions to drive forward this important work.

Competing interests: David Wrigley is BMA chief officer and deputy chair of the BMA UK Council. Latifa Patel is chief officer and board member at the BMA: trustee of BMA Charities; council member of the UK Health Alliance on Climate Change and WMA; member of the RCPCH; employed by St Helens and Knowsley

Provenance and peer review: Not commissioned; not externally peer reviewed.

Department of Health and Social Care. DHSC: gender pay gap report and data 2021. Gov.uk. 27 January 2022. https://www.gov.uk/government/publications/dhsc-gender-pay-gap-report-and-data-2021/dhsc-gender-paygap-report-and-data-2021\#introduction

2 Sexism in medicine. British Medical Association. 2021 https://www.bma.org.uk/media/4487/sexism-in-medicine-bma-report.pdf is the right time for the implementation panel to stop lead employer. 
3 Mend the Gap: The Independent Review into Gender Pay Gaps in Medicine in England. Department of Health and Social Care. December 2020. https://assets.publishing.service.gov.uk/government/uploads/system/uploads/attachment_data/file/944246/Gender_pay_gap_in_medicine_review.pdf 